\title{
The Behavior of Metal Jet in the In-Rotating-Water Spinning Method*
}

\author{
By Yoshio ABE,** Ken-ichi MIYAZAWA, ${ }^{* *}$ Masakazu NAKAMURA** \\ and Tetsuro OHASHI***
}

\begin{abstract}
Synopsis
To understand the formation of rapidly solidified metal wire directly from the molten state by the In-Rotating-Water Spinning Method, some experiments have been done by the use of $\mathrm{Pb}-\mathrm{Sb}$ alloy. In the experiments, the behavior of molten metal jet in rotating water was directly observed to reveal that the jet orbit closely relates with the cross-sectional shape, longitudinal shape and continuity of wires obtained.

Under the experimental conditions adopted in this study, it has been confirmed that the apparent properties of the wire are influenced mainly by both the jet velocity and the water-drum velocity. With increasing ratio of the jet velocity to the water velocity, molten metal jet penetrates deeper into rotating water. The range showing the optimum conditions for the production of long-continued straight wire has also been determined.

Furthermore, the theoretical methods to estimate the jet velocity at the nozzle outlet and the jet orbit in water have been developed. The jet velocity of molten metal can well be predicted by the use of the equivalent nozzle orifice diameter obtained from the water experiment on the characteristics of nozzle. The calculated jet orbit agrees well with the observed results.
\end{abstract}

Key words: rapid solidification; melt spinning; jet; wire; lead-antimony alloy; direct casting; mathematical modeling.

\section{Introduction}

Direct casting of metal thin wire from the molten state is attractive in the light of simplification of the wire production process and improvement in the properties of wire due to rapid solidification. Hitherto, various methods have been proposed for direct casting of metal wire, filament or fiber; namely free flight melt spinning, ${ }^{1)}$ chill block melt spinning, ${ }^{1}$ Taylor process $^{2)}$ (or glass-coated melt spinning ${ }^{3)}$ ), crucible melt extraction and pendant drop melt extraction, ${ }^{4)}$ Kavesh process ${ }^{5)}$ and In-Rotating-Water Spinning process. ${ }^{6)}$

Among the methods proposed on the direct casting of metal wire the In-Rotating-Water Spinning developed by Ohnaka et al. ${ }^{6)}$ seems to be superior to the production of long-continued and circular wire under a rapid cooling rate. Glassy or crystalline metal wire prepared by this process has been investigated especially on the magnetic and mechanical properties, and on the findings of new materials. ${ }^{7-12)}$

In the process, a jet of molten metal is plunged into water in a rotating drum. Hydrodynamic behavior of the molten metal jet in rotating water is expected to affect the properties of wire. However, less information is avairable on the effects of the process variables on the formability of wire ${ }^{6)}$ and the behavior of jet in water is not known. Thus, in this study, the relationship among the process variables, the behavior of jet in rotating water and the apparent properties of wires has been studied through the observation and theoretical analysis of the jet.

\section{Experimental}

A schematic representation of the experimental apparatus is shown in Fig. 1. In the experiment, at first, a water layer is formed inside the rotating drum by the centrifugal force. A jet of molten metal is ejected by argon through an orifice of quartz nozzle into the rotating water layer, $15 \mathrm{~mm}$ in thickness. The orifice diameter of the nozzle used is ranged from 100 to $140 \mu \mathrm{m}$. The diameter and width of the drum are $300 \mathrm{~mm}$ and $100 \mathrm{~mm}$, respectively. The front wall of drum is made of transparent acrylic resin plate, through which the behavior of molten metal jet in water can be observed. Hypoeutectic alloy (alloy A) of $\mathrm{Pb}-4.2 \mathrm{wt} \% \mathrm{Sb}$ and eutectic alloy (alloy $\mathrm{B}$ ) of $\mathrm{Pb}-11.1 \mathrm{wt} \% \mathrm{Sb}$ were used in the experiments.

In this study, the experiment was done mainly by changing the jet and drum velocities, in the ranges of $4 \sim 10 \mathrm{~m} / \mathrm{s}$ and $3 \sim 10 \mathrm{~m} / \mathrm{s}$, respectively. Superheat of molten metal in the nozzle was kept at $150 \mathrm{~K}$. The distance between the nozzle outlet and water surface was nearly $5 \mathrm{~mm}$ and the incidence angle of jet to the water surface was $60^{\circ}$.

\section{Theoretical Analysis}

\section{Velocity of Liquid Jet}

Figure 2 shows a schematic view of the nozzle used in this study. Prediction of the jet velocity is important for the production of long-continued wire with good quality.

From the mechanical energy balance between the meniscus of molten metal and nozzle outlet, the rela-

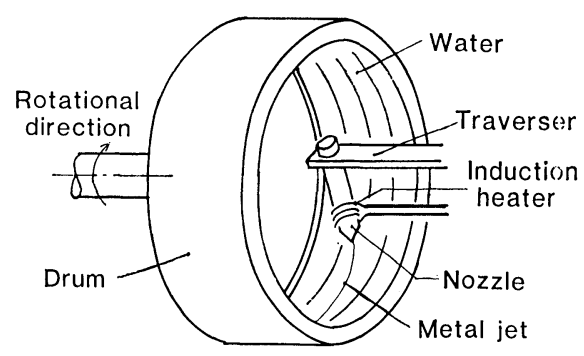

Fig. 1. A schematic representation of the experimental apparatus.

* Based on the paper presented to the 111th ISIJ Meeting, April 1986, S766, at The University of Tokyo in Tokyo. Manuscript received on March 31, 1987; accepted in the final form on July 3, 1987. (C) 1987 ISIJ

** $\quad$ R \& D Laboratories-I, Nippon Steel Corporation, Ida, Nakahara-ku, Kawasaki 211.

*** Central R \& D Bureau, Nippon Steel Corporation, Otemachi, Chiyoda-ku, Tokyo 100. 
tionship between the flow rate and ejection pressure is given as follows;

$$
Q^{2}=\left(\pi d_{n}^{2} / 4\right)^{2} \cdot(2 / \rho) \cdot C_{f}^{2} \cdot\left(P_{n}+\rho g h-2 \sigma / d_{n}\right)
$$

where, $Q$ : the volumetric flow rate

$d_{n}:$ the nozzle orifice diameter

$\rho$ : the density

$g$ : the acceleration of gravity

$h$ : the head of molten metal

$\sigma:$ the surface tension

$P_{n}:$ the ejection pressure

$G_{f}$ : the flow coefficient.

The flow coefficient $C_{f}$ is given by Eq. (2).

$$
C_{f}=\left(1+K+\lambda l_{n} / d_{n}\right)^{-1 / 2}
$$

where, $\lambda:$ the friction factor for the conical part of nozzle in Fig. 2

$K:$ the friction loss factor

$l_{n}$ : the height of conical part of nozzle.

The friction factor $\lambda$ is expressed as follows:

For laminar flow: $\lambda=(64 / R e) \cdot \beta$

For turbulent flow : $\lambda=\left(0.32 / R e^{0.25}\right) \cdot \beta$

where,

$$
\beta=\frac{d_{n}}{l_{n}} \cdot \frac{1}{3 \tan (\theta / 2)} \cdot\left[1-\frac{1}{\left\{1+l^{\prime} \cdot \tan (\theta / 2)\right\}^{3}}\right]
$$

$l^{\prime}=l_{n} / d_{n}$

$R e=\rho V_{J} d_{n} / \mu$, the Reynolds number

$\mu:$ the viscosity

$\theta: \quad$ the cone angle of nozzle.

The friction factor for the conical part of nozzle ( $\lambda$ ) in Eqs. (3) and (4) can be obtained by following the derivation of the flow velocity and the friction factor for the straight tube. ${ }^{13)}$

Jet velocity is obtained from Eq. (1) as follows;

$$
V_{J}=Q /\left(\pi d_{n}^{2} / 4\right)
$$

The jet velocity depends mainly on the ejection pressure and orifice diameter of nozzle. Accurate measurement of the orifice diameter is practically difficult. Therefore, in this study, the orifice diameter was estimated so as to satisfy Eq. (1) for water jet. With the estimated orifice diameter $\left(d_{\mathrm{cal}}\right)$, the jet velocity for molten metal is calculated from Eq. (7).

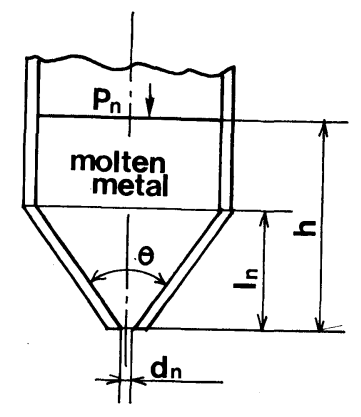

Fig. 2. A schematic view of the nozzle used.

$$
V_{J, \text { cal }}=C_{f} \cdot\left\{(2 / \rho) \cdot\left(P_{n}+\rho g h-2 \sigma / d_{\text {cal }}\right)\right\}^{1 / 2}
$$

On the other hand, the jet velocity can be obtained on the basis of experimental data as follows;

(a) $V_{J}$ derived from the observed mass flow rate

$$
V_{J, \mathrm{obs}}^{\mathrm{I}}=w /\left(\Delta t \cdot \rho_{l} \cdot \pi d_{n}^{2} / 4\right)
$$

(b) $V_{J}$ derived from the observed diameter of solidified wire

$$
V_{J, \mathrm{obs}}^{\mathrm{II}}=w /\left(\Delta t \cdot \rho_{s} \cdot \pi D_{w}^{2} / 4\right)
$$

where, w: the mass of molten metal

$\Delta t:$ the ejection time

$\rho_{s}, \rho_{l}:$ the densities of solid and liquid metals, respectively

$D_{w}$ : the effective mean diameter of solidified wire

$D_{w}=\{($ the major diameter $) \times($ the minor diameter) $\}^{1 / 2}$.

In the experiment, a high speed video recorder (200 frames/s) was used to measure the ejection time $(\Delta t)$, which was usually $5 \sim 6 \mathrm{~s}$ for the ejection of molten metal of about $5 \mathrm{~g}$. The mass of ejected metal $(w)$ can precisely be measured by weighing after the ejection. Thus, the errors in the measurements of $w$ and $\Delta t$ are thought to be small for calculations of the jet velocities from Eqs. (8) and (9). Strictly speaking, there are two periods of lower jet velocity at the initiation and the end of ejection, because of the delay of pressure in the nozzle. The length of these periods were roughly $0.4 \mathrm{~s}$ in total for the ejection pressure of 0.3 0.5 MPa. Thus, Eqs. (8) and (9) give slightly smaller jet velocities than the real steadystate one.

\section{Orbit of Liquid Jet in Rotating Water}

In the In-Rotating-Water Spinning Method, it is important to understand the behavior of liquid metal jet in flowing water. Although the deformation of liquid metal jet in hypersonic gas flow has been theoretically analyzed by Catton et al. ${ }^{14)}$ the analysis for liquid metal jet in water flow is not reported yet.

Figure 3 shows a schematic representation of the jet orbit in rotating water. In this figure, a liquid metal jet is plunging into rotating water with an incidence angle of $\alpha_{0}$ to the water surface. The orbit of liquid metal jet is continuously deformed by the drag force of water to the direction of water flow.

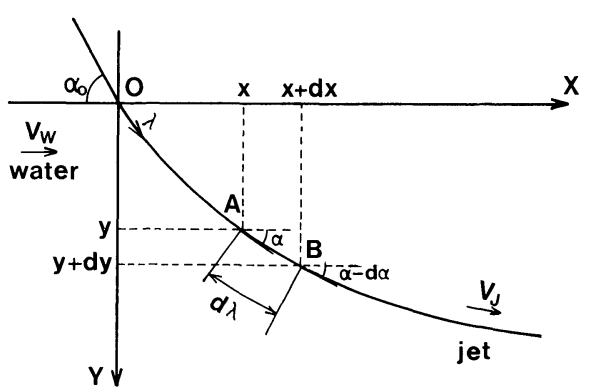

Fig. 3. A schematic representation of the jet orbit in rotating water. 
During the deformation, the jet of molten metal is cooled and solidifies at a position. After the solidification, no deformation of the solidified wire due to water flow may take place.

Under the assumptions that the cross section of wire is circular and the diameter $(D)$ is kept constant, making the momentum balance of jet in a section from $x$ to $x+d x$ yields the change in the component of momentum normal to the jet orbit $\left(\Delta F_{1}\right)$ as follows;

$$
\Delta F_{1}=-\rho_{J} V_{J}^{2}\left(\pi D^{2} / 4\right) \cdot d \alpha
$$

where, $\rho_{J}$ : the density of molten metal

$\alpha$ : the angle of jet orbit to the water surface.

On the other hand, the component of the drag due to water flow normal to the jet orbit $\left(F_{2}\right)$ is given by Eq. (11) on the basis of the Newton's law for the pressure of fluid flow. ${ }^{15)}$

$$
F_{2}=C_{d} \cdot\left\{\rho_{\mathrm{w}}\left(V_{\mathrm{w}} \sin \alpha\right)^{2} / 2\right\} \cdot D d \lambda
$$

where, $C_{d}$ : the apparent drag coefficient

$\rho_{\mathrm{w}}:$ the density of water

$V_{\mathrm{w}}$ : the velocity of water

$\lambda$ : the curvilinear coordinate along jet orbit.

If $\Delta F_{1}$ is equalized to $F_{2}$, it yields the following differential equation for the deformation of jet orbit.

$$
d \lambda=-\frac{\rho_{J} V_{J}^{2} \cdot \pi D^{2} / 2}{C_{d} \rho_{\mathrm{w}} V_{\mathrm{w}}^{2} \cdot D} \cdot \frac{d \alpha}{\sin ^{2} \alpha}
$$

Substituting geometrical relations such as $d \lambda=d x /$ $\cos \alpha$ and $d \lambda=d y / \sin \alpha$, respectively, and solving the resultant equations under the boundary condition of $\alpha=\alpha_{0}$ at $x=0$ and $y=0$, it yields the jet orbit as follows;

$$
\begin{aligned}
& x=K_{1}\left(\frac{1}{\sin \alpha}-\frac{1}{\sin \alpha_{0}}\right) \ldots \ldots \ldots \ldots \ldots \ldots \ldots \ldots \ldots \ldots \ldots \ldots \\
& y=\frac{K_{1}}{2}\left\{\ln \left(\frac{1+\cos \alpha}{1-\cos \alpha}\right)-\ln \left(\frac{1+\cos \alpha_{0}}{1-\cos \alpha_{0}}\right)\right\}
\end{aligned}
$$

where, $\quad K_{1}=\frac{\pi D}{2 C_{d}} \cdot \frac{\rho_{J} V_{J}^{2}}{\rho_{\mathrm{w}} V_{\mathrm{w}}^{2}}$

From the investigation of Eqs. (13) and (14), it has been found that the jet penetrates deeper in water with increasing $K_{1}$ in Eq. (15), namely with increasing jet diameter $(D)$, the ratio of jet density to that of water $\left(\rho_{J} / \rho_{\mathrm{w}}\right)$ and the ratio of jet velocity to that of water $\left(V_{J} / V_{\mathrm{w}}\right)$.

\section{Results and Discussion}

\section{Estimation of Jet Velocity}

The relationship observed between the ejection pressure and the flow rate of water system is shown in Fig. 4. The square of the flow rate of water has been found to have linear relationship with the ejection pressure for every nozzle used, in accordance with the theoretical relation given in Eq. (1). The gradient of the straight line in Fig. 4 becomes larger with increasing diameter of nozzle orifice.

Figure 5 shows the measured nozzle orifice diameter $\left(d_{n}\right)$, compared with the diameter calculated for the laminar flow $\left(d_{\text {cal }}\right)$ from Eq. (1) and for the observed flow rate for water system. The calculated diameter is smaller than the measured one. This may be attributed to the overestimation of observed orifice diameter due to chipping at the edge of nozzle outlet.

In this experiment, the flow at nozzle orifice can be assumed to be laminar because the Reynolds number estimated is in the range of 2000 to 5000 . The nozzle orifice diameter calculated for the turbulent flow is smaller than that of laminar flow by a few micron. This means that each calculated diameter can be regarded as the same. Therefore, the orifice diameter calculated for the laminar flow was used as $d_{\text {cal }}$ in the following discussion.

Figure 6 shows the relationship observed between the square of the flow rate of molten $\mathrm{Pb}-\mathrm{Sb}$ alloy and the ejection pressure. In these experiments, the nozzles were repeatedly used. The orifice diameter was observed to be unchanged by repetition of the ejec-

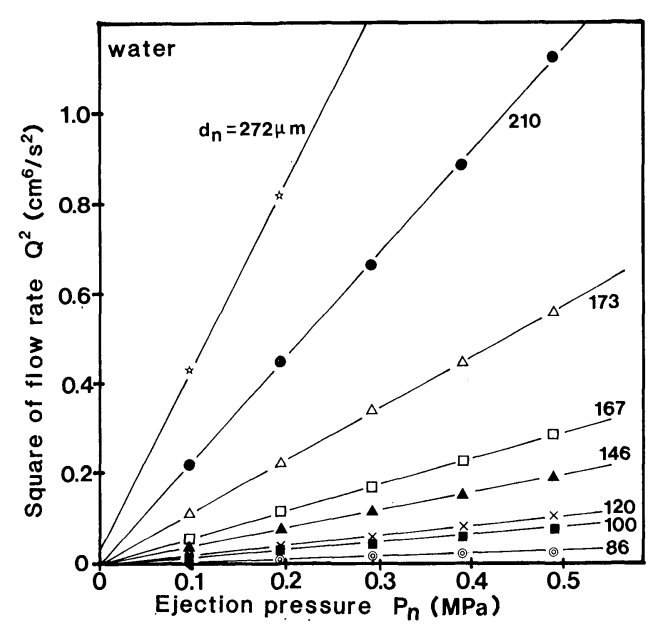

Fig. 4. Observed relationship between the ejection pressure and the flow rate for water system.

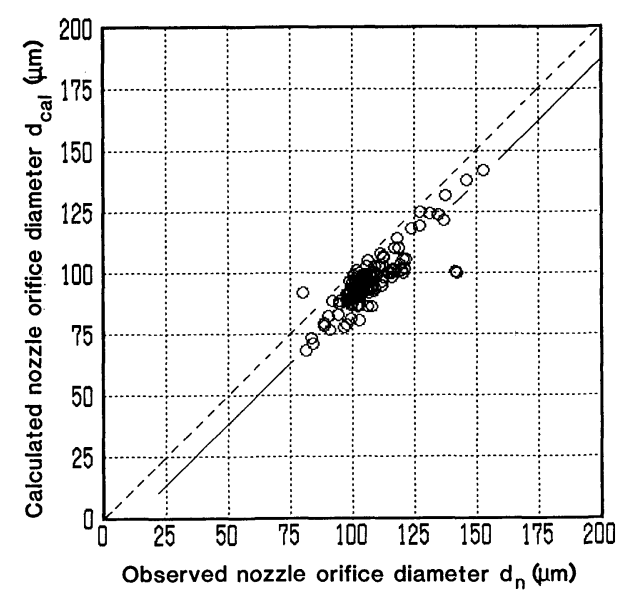

Fig. 5. Comparison between the measured nozzle orifice diameter and that calculated for the case of laminar flow. 
tion of molten metal and cleaning. In Fig. 6, a linear relationship between the square of the flow rate and the ejection pressure is also found for the molten metal in the same manner as that of water.

Figure 7 shows the observed jet velocities for $\mathrm{Pb}-$ $\mathrm{Sb}$ alloys, compared with the calculated jet velocities. In Figs. 7(a) and 7(c), the calculated jet velocity is slightly larger than the velocity obtained from the measured flow rate $\left(V_{J, \mathrm{obs}}^{\mathrm{I}}\right)$, while in Figs. $7(\mathrm{~b})$ and

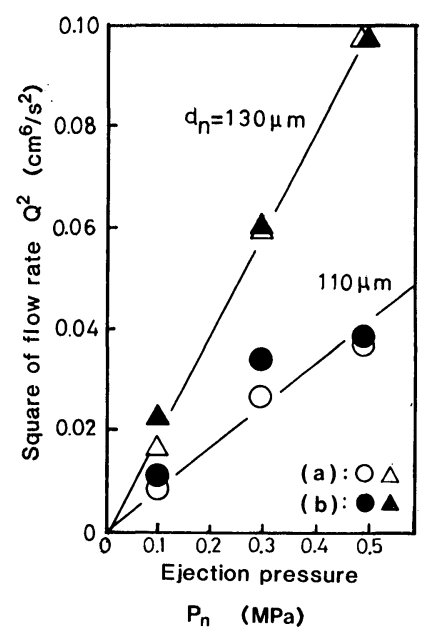

$\begin{array}{ll}\text { (a) } \mathrm{Pb}-4.2 \mathrm{wt} \% \mathrm{Sb} & \text { (b) } \mathrm{Pb}-11.1 \mathrm{wt} \% \mathrm{Sb}\end{array}$

Fig. 6. Observed relationship between the square of the flow rate of molten $\mathrm{Pb}-\mathrm{Sb}$ alloy and ejection pressure.
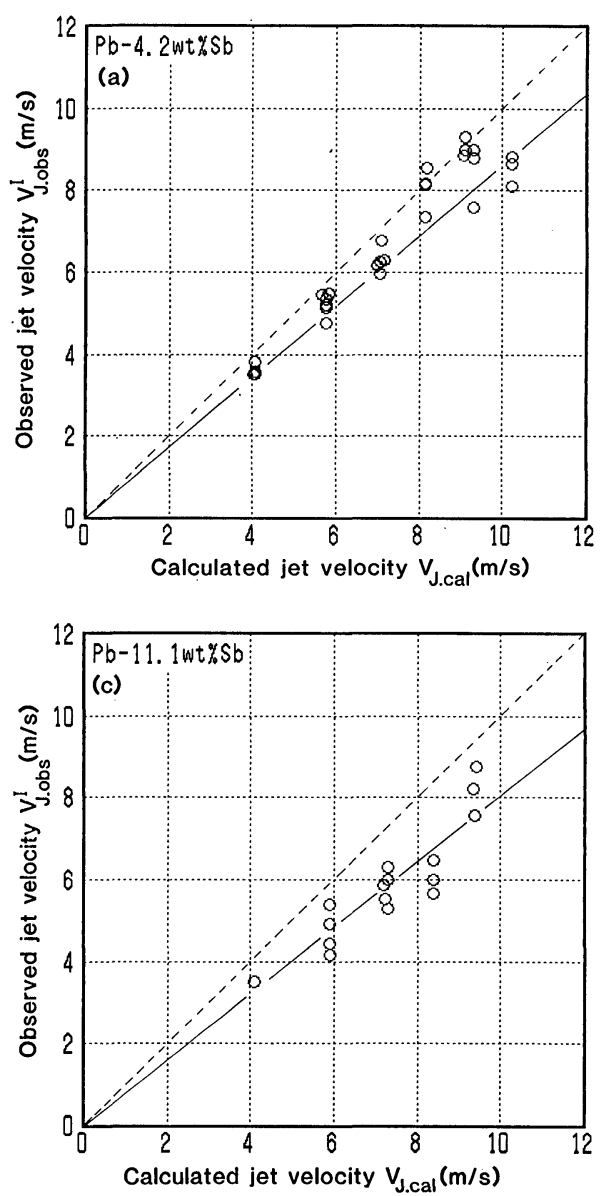

7 (d) the jet velocity obtained from the observed diameter of solidified wire $\left(V_{J, \text { obs }}^{\mathrm{II}}\right)$ is much smaller than the calculated one. The relation among these jet velocities is expressed as follows;

$$
V_{J, \mathrm{obs}}^{\mathrm{II}}<V_{J, \mathrm{obs}}^{\mathrm{I}}<V_{J, \mathrm{c}}
$$

As can be seen from Eqs. (7) and (8), the slight difference between $V_{J \text {, cal }}$ and $V_{J}^{\mathrm{I} \text {, obs }}$ comes mainly from the difference in the nozzle orifice diameter adopted. The calculated nozzle orifice diameter smaller than the observed one is used for $V_{J \text {, cal }}^{\mathrm{I}}$ in Eq. (7). On the other hand, the observed diameter is used for $V_{J, \text { obs }}^{\mathrm{I}}$ in Eq. (8). So, $V_{J \text {, cal }}$ becomes higher than $V_{J, \text { obs }}^{\mathrm{I}}$, as expressed by Eq. (16). When the same nozzle orifice diameter is used for calcula tions of $V_{J \text {,eal }}$ and $V_{J, \text { obs }}^{\mathrm{I}}$, better agreement is obtained between $V_{J \text {, cal }}$ and $V_{J, \text { obs }}^{\mathrm{I}}$. On the other hand, the jet velocity obtained from the observed diameter of wire $\left(V_{J}^{\mathrm{II}}\right.$ obs $)$ is much scattered in Figs. 7 (b) and $7(d)$. This results from the rough estimation of the effective mean diameter of solidified wire $\left(D_{w}\right)$. The wire diameter is usually smaller than the observed nozzle orifice diameter. Thus, $V_{J}^{\mathrm{II} \text { obs }}$ obtained by the use of the wire diameter is smaller than $V_{J, \text { obs }}^{\mathrm{I}}$, as shown in Eq. (16).

\section{Effects of Operational Variables on Apparent Properties of Rapidly Solidified Wire}

Figure 8 shows the surface appearance of the wire
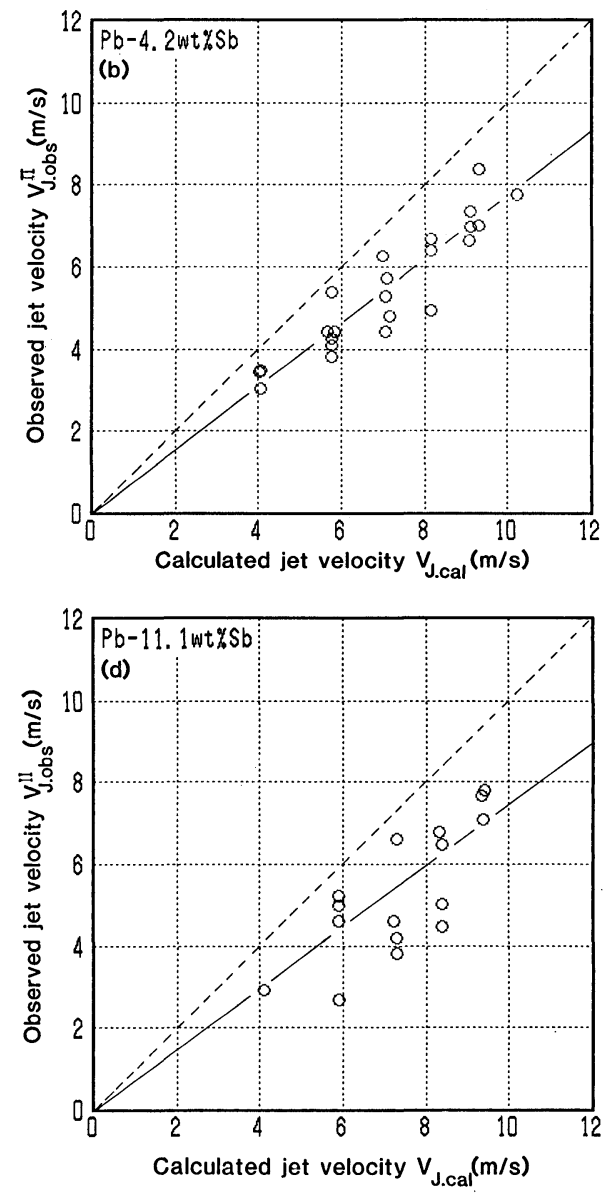

Fig. 7. Comparisons between the observed and the calculated jet velocities for $\mathrm{Pb}-\mathrm{Sb}$ alloy. 


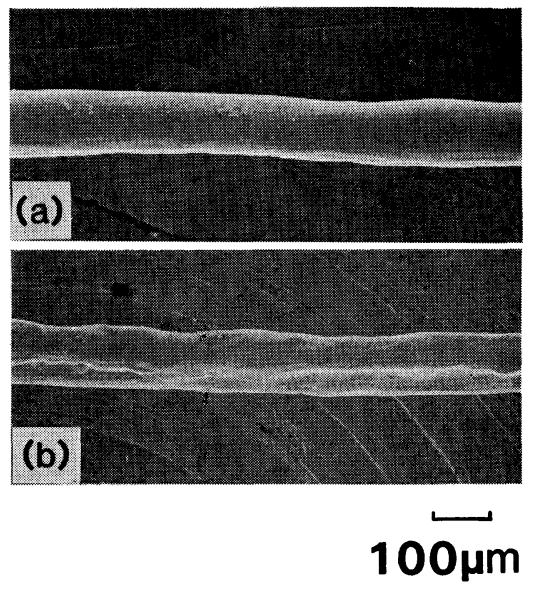

(a) $\mathrm{Pb}-4.2 \mathrm{wt} \% \mathrm{Sb}$

(b) $\mathrm{Pb}-11.1 \mathrm{wt} \% \mathrm{Sb}$

Fig. 8. Appearance of wires obtained.

prepared in this study. The surface of the wire is not smooth and microscopic cracks are often observed. The wire of the eutectic $\mathrm{Pb}-\mathrm{Sb}$ alloy (B) shows more irregular surface than that of hypoeutectic alloy (A).

A cross-sectional view of the wire obtained is shown in Fig. 9. As the drum velocity $\left(V_{D}\right)$ increases, the cross-sectional shape changes from circular to oblate.

Rapidly solidified wires of iron-based alloys $8,9,12)$ and $\mathrm{Pd}$ alloys ${ }^{6,7)}$ have been reported to be circular and smooth. This comes mainly from much lower surface tension of molten $\mathrm{Pb}-\mathrm{Sb}$ alloy than those of iron-base alloy and $\mathrm{Pd}$ alloy. In fact, the surface tension of $\mathrm{Pb}-\mathrm{Sb}$ alloy is roughly three times as small as that of Fe alloy. ${ }^{16)}$

The effect of the drum and jet velocities on the length of wire, over which the molten metal jet is not broken in water, is shown in Fig. 10. There exists an experimental condition where the wire becomes longer than $1 \mathrm{~m}$, being expressed as $1.0<V_{J} / V_{D}<1.4$ and $1.0<V_{J} / V_{D}<1.6$ for the alloys $\mathrm{A}$ and $\mathrm{B}$, respectively, where $V_{D}$ is the drum velocity.

Ohnaka et al. ${ }^{6)}$ reported that the experimental condition to produce long-continued wire is expressed as $1<V_{J} / V_{D}<1 / \cos \alpha_{0}$, where $\alpha_{0}$ is the incidence angle of jet to the water surface. The region satisfying the condition is described as the region between the broken line and the straight line of $V_{J}=V_{D}$ in Figs. 10 (a) and 10(b). The condition for long-continued wire obtained in this study roughly agrees with that proposed by Ohnaka et al.

As to the longitudinal shape of the wire obtained, it has been found that the shape changes from straight to wavy with increasing ratio of the jet velocity to the drum (water) velocity. The condition where the wire becomes straight can be expressed by $V_{J} / V_{D}<1.2$ for both alloys (A and B) used. When the jet velocity is much higher than the water velocity, the molten metal jet becomes to bend in water and eventually breaks before the solidification of metal jet to a wavy wire, because the solidified wire is considered to finally rotate with the same velocity as that of water. On the other hand, when the metal jet velocity is

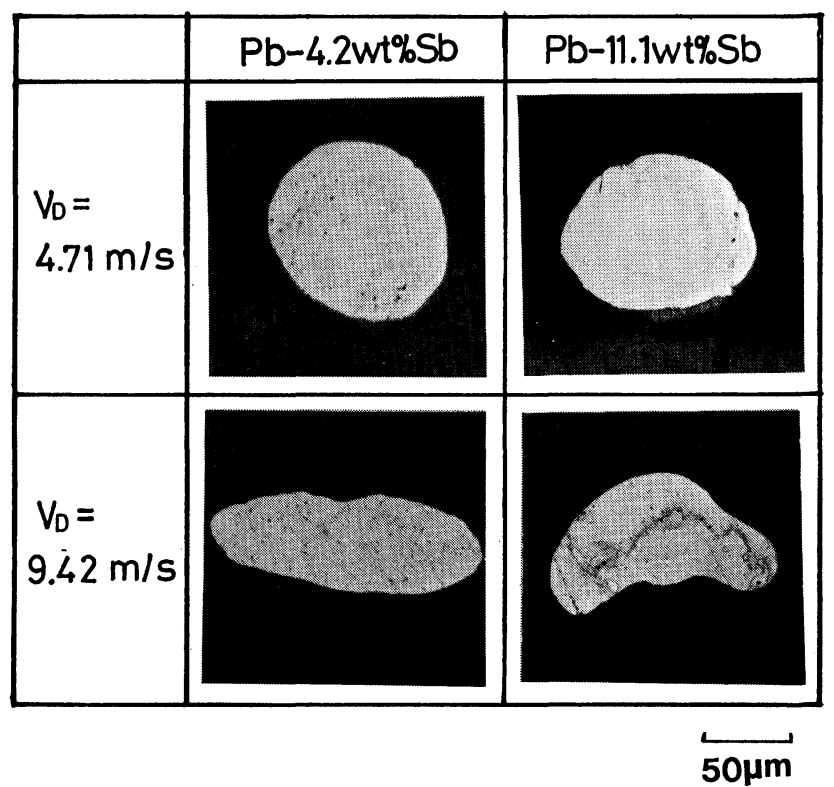

Fig. 9. Cross-sectional views of solidified wire.
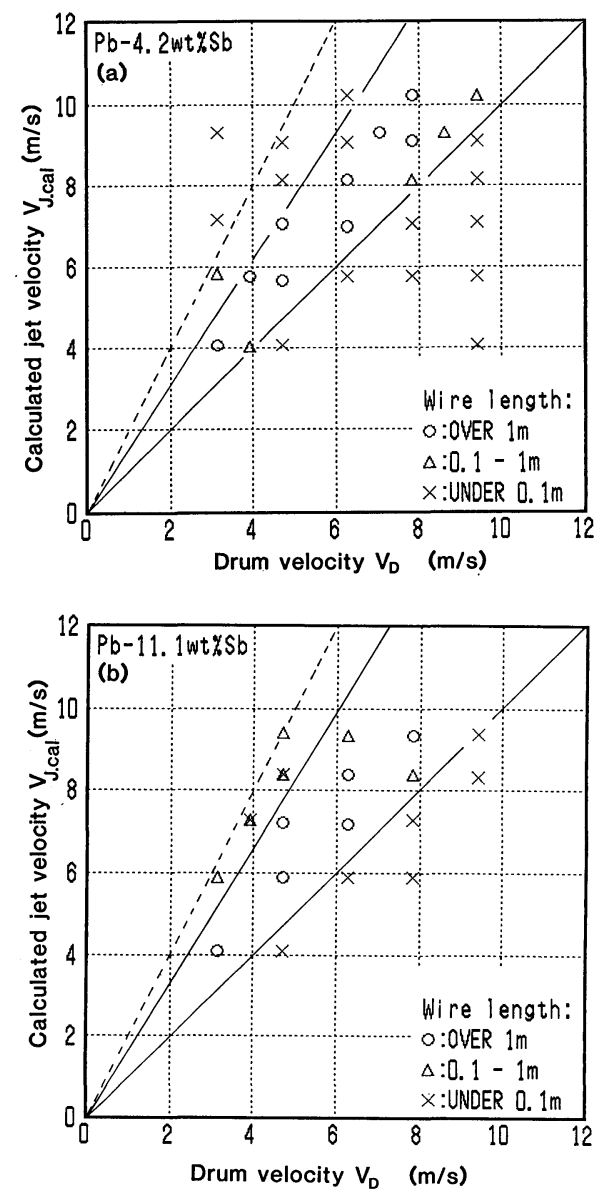

Fig. 10. Effect of the drum and the jet velocities on the length of solidified wire.

lower than the water velocity, the molten metal jet is drawn out by water and breaks intermittently.

Therefore, with increasing ratio of the jet velocity to the water velocity, the formation of wire in water changes from the discontinued straight wire, the longcontinued straight one to the discontinued wavy one. 
The condition to obtain the long-continued straight wire is expressed by $1.0<V_{J} / V_{D}<1.2$ for the $\mathrm{Pb}-\mathrm{Sb}$ alloy used.

Figure 11 shows the effect of the drum velocity on the circularity of the cross section of wire. The circularity $(\varepsilon)$ is defined as the ratio of the minor to the major diameters of wire. It can be seen from Fig. 11 that the circularity becomes approximately unity, namely the cross section of wire becomes more circular, as the drum (water) velocity decreases. The effect of the jet velocity on the circularity of wire is not clear under the experimental condition adopted in this study. The circularity of wire may depend on the dynamic pressure of water, the diameter and the surface tension of molten metal jet. Ohnaka et al. $\left.{ }^{6}\right)$ suggested that the circularity of lead wire is proportional to $\left(V_{\mathrm{w}} \sin \alpha_{0}\right)^{2}$, where $V_{\mathrm{w}} \sin \alpha_{0}$ is the component of water velocity normal to the jet axis. The relation is observed in Fig. 11 to be also valid for $\mathrm{Pb}-\mathrm{Sb}$ alloy.

\section{Behavior of Molten Metal Jet in Rotating Water}

Figure 12 is a photograph showing the metal jet plunging into the rotating water layer. The jet is observed to be smoothly curved by the water flow into the direction parallel to the water surface. A valleylike cavity is formed behind the molten metal jet and vanishes in several centimeters from the plunging point of jet. After solidification of the jet, the solidified wire accumulates inside of the rotating drum.

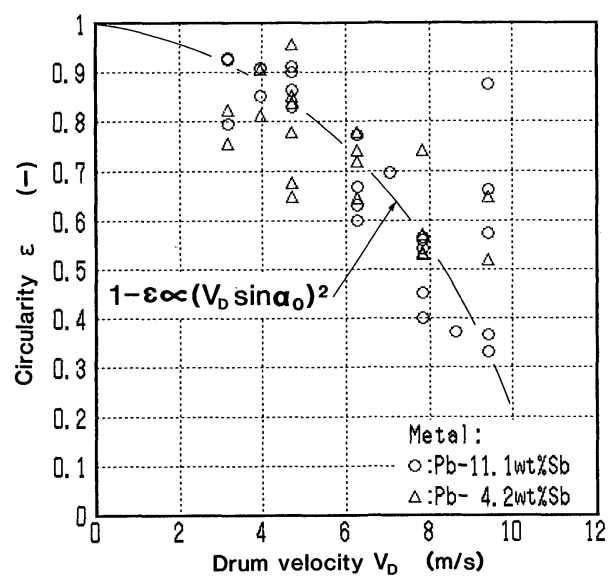

Fig. 11. Effect of the drum velocity on the circularity of solidified wire.

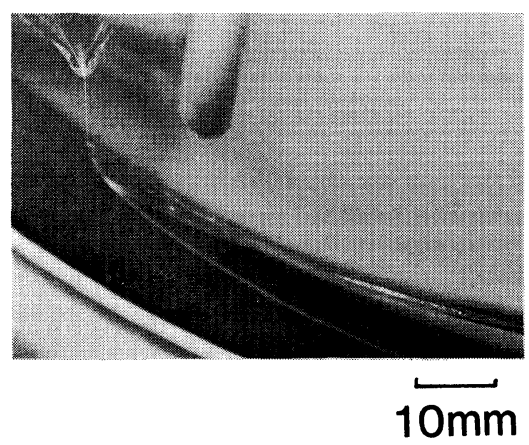

Fig. 12. Observed behavior of metal jet plunging into the rotating water layer.
As can be seen from Fig. 12 showing the molten metal jet in rotating water, a cavity is formed behind the molten metal jet. In this case, the drag due to water flow may be different from the case without the cavity. Equation (11) is derived on the basis of the Newton's law for the pressure of fluid flow, ${ }^{15)}$ being assumed that the fluid composed of particles causes non-elastic collision with the surface of an object to generate the pressure on the object surface. As far as the Newton's law is applied, Eq. (11) is obtained regardless of the existence of the cavity behind the jet. The validity of the Newton's law is fairly well for the gas flow, but is dependent on the Reynolds number for the liquid flow, namely it becomes better as the Reynolds number becomes larger. Therefore, an apparent drag coefficient was introduced in Eq. (11) to correct the discrepancy between the Newton's law and the present result for water. As can be seen from Fig. 15, the apparent drag coefficient is approximately unity and very close to the drag coefficient for a cylinder which is standing normal to the fluid flow. This means that the application of the Newton's law, namely Eq. (11), is approximately valid for the jet with cavity.

The effect of drum velocity on the observed jet orbit is shown in Fig. 13. The jet penetrates deeper into water with decreasing drum velocity. It can also be seen from Fig. 13 that the cross-sectional shape of wire becomes more circular as the jet penetrated deeper in the water layer. It has been also found

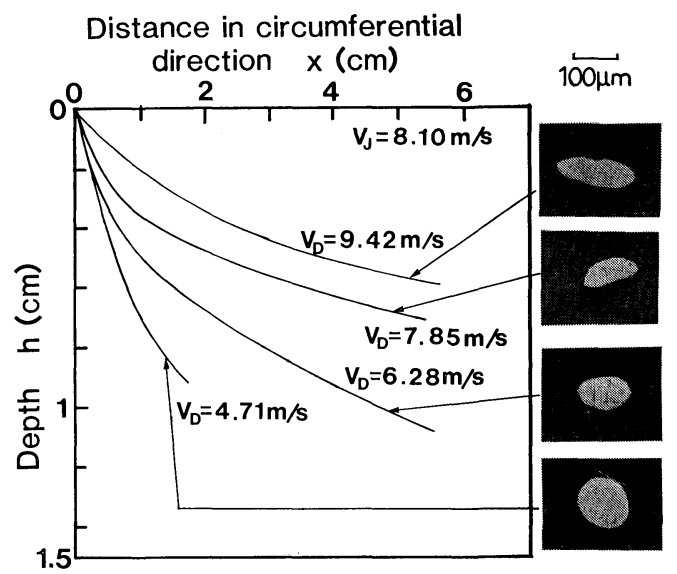

Fig. 13. Effects of drum velocity on the observed jet orbit in rotating water and the cross section of wire.

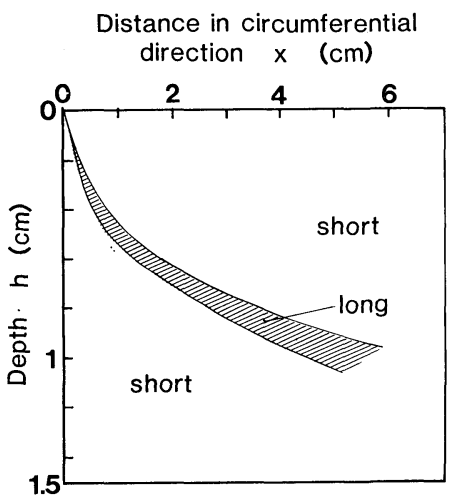

Fig. 14. The shape of jet orbit for long-continued wire. 


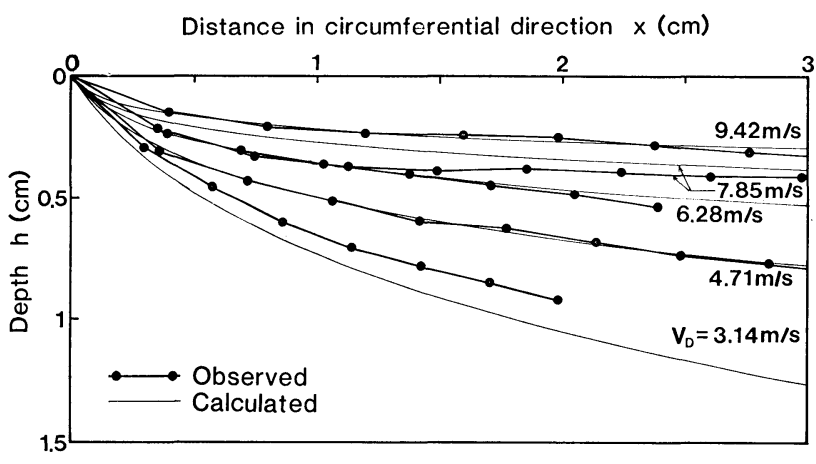

Fig. 15. Comparison between the observed and the calculated jet orbits in rotating water.

that the jet penetrates deeper into water with increasing jet velocity.

Figure 14 shows the shape of the jet orbit in which a long-continued wire can be produced without breakup of molten metal jet. This shape of the jet orbit can be obtained under the condition of $1.0<V_{J} / V_{D}<$ 1.2. Outside of the region, the jet is often broken out by the bending or by the drawing in water.

Figure 15 shows the observed jet orbit in water, compared with the calculated jet orbit. The conditions adopted for calculations are as follows; $V_{J}=7$ $\mathrm{m} / \mathrm{s}, \quad D=110 \mu \mathrm{m}, \rho_{\mathrm{w}}=1.0 \mathrm{~g} / \mathrm{cm}^{3}, \rho_{J}=7.0 \mathrm{~g} / \mathrm{cm}^{3}$, and $\alpha_{0}=60^{\circ}$. In calculations, the apparent drag coefficient was kept constant at $C_{d}=1.3$. This value of $C_{d}$, which was selected so as to get better agreement between the calculated and the observed jet orbits, is almost the same as the drag coefficient for a cylinder which is standing normal to the fluid flow $\left(C_{d} \approx 1.5 \sim\right.$ 1.0 for $R e=300 \sim 1400$ corresponding to the present experimental condition, where $\left.R e=\rho_{\mathrm{w}} V_{\mathrm{w}} D / \mu_{\mathrm{w}}\right) .{ }^{17)} \quad$ It can be seen from Fig. 15 that the calculated jet orbit agrees fairly well with the observed one. This means that the component of the dynamic pressure of water normal to the jet axis mainly determines the jet orbit, as discussed in the theoretical analysis of jet orbit.

\section{Conclusions}

In order to understand the formation of rapidly solidified wire from a jet of molten metal in the InRotating-Water Spinning Method, some experiments have been done by the use of $\mathrm{Pb}-\mathrm{Sb}$ alloys. Furthermore, the jet velocity at the nozzle outlet and the jet orbit in rotating water have been theoretically analyzed. The results obtained are summarized as follows:

(1) The molten metal jet ejected through a nozzle orifice plunges deeper into rotating water with increasing jet velocity and decreasing water velocity, following that the appearance of wire changes from discontinued straight wire, long-continued straight one to discontinued wavy one. The cross section of the wire obtained becomes more circular with decreasing water velocity.

(2) The optimum experimental condition for long-continued straight wire is expressed by $1.0<$ $V_{J} / V_{D}<1.2$ for both $\mathrm{Pb}-4.2 \mathrm{wt} \% \mathrm{Sb}$ and $\mathrm{Pb}-11.1 \mathrm{wt} \%$ $\mathrm{Sb}$ alloys, where $V_{J}$ and $V_{D}$ are the jet velocity and the water-drum velocity, respectively.

(3) The velocity of molten metal jet can well be estimated by the use of the equivalent nozzle orifice diameter obtained from the water experiment on the characteristics of nozzles and the theoretical equations derived in the current work.

(4) The orbit of molten metal jet in rotating water closely relates to the cross-sectional shape, longitudinal shape, and the length of wire. The calculated jet orbit agrees well with the observed results.

\section{REFERENCES}

1) R. E. Maringer and C. E. Mobley: Wire J., 12 (1979), 70.

2) G.W.F. Pardoe, E. Butler and D. Gelder: J. Mater. Sci., 13 (1978), 786.

3) T. Goto: Mater. Sci. Eng., 59 (1983), 251.

4) R. E. Maringer and G. E. Mobley: J. Vac. Sci. Technol., 11 (1974), 1067.

5) S. Kavesh: AIChE J., Symp. Series, 74 (1978), No. 180, 1.

6) I. Ohnaka, T. Fukusako and T. Ohmichi: J. Jpn. Inst. Met., 45 (1981), 751.

7) T. Masumoto, I. Ohnaka, A. Inoue and M. Hagiwara: Scr. Metall., 15 (1981), 293.

8) A. Inoue, M. Hagiwara and T. Masumoto: J. Mater. Sci., 17 (1982), 580.

9) M. Hagiwara, A. Inoue and T. Masumoto: Metall. Trans. $A$, 13A (1982), 373.

10) A. Inoue, $Y$. Masumoto, N. Yano, A. Kawashima, K. Hashimoto and T. Masumoto: J. Mater. Sci., 20 (1985), 97.

11) A. Inoue, N. Yano and T. Masumoto: Trans. Iron Steel Inst. Jpn., 25 (1985), 1069.

12) T. Ichiryu, I. Ohnaka, H. Tateoka and I. Yamauchi: J. Jpn. Inst. Met., 50 (1986), 927.

13) R. B. Bird, W. E. Stewart and E. N. Lightfoot: Transport Phenomena, John Wiley \& Sons, New York, (1960), 42 \& 183.

14) I. Gatton, D. E. Hill and R. P. McRae: AIAA J., 6 (1968), 2084.

15) B. Fujimoto: Ryutai Rikigaku (Fluid Dynamics), Yohkendo, Tokyo, (1964), 123 \& 277.

16) L. E. Murr: Interfacial Phenomena in Metals and Alloys, Addison-Wesley, Reading, (1975), 101.

17) S. Ito et al.: Kagaku Kogaku Benran, 3rd Ed., ed. by S. Fujita et al., Maruzen, Tokyo, (1968), 112. 